\title{
Gel Determination in the Radiation Grafting of 4-Vinylpyridine to Polyethylene
}

\author{
M. I. Aly, ${ }^{*}$ S. A. Hegazy, ${ }^{*}$ and A. M. RABIE** \\ *National Centre for Radiation Research and Technology, \\ Madinet Nasr, Cairo, Egypt. \\ **Chemistry Department, Faculty of Science, \\ Ain Shams University, Cairo, Egypt.
}

(Received November 2, 1978)

\begin{abstract}
The gel copolymer formed when monomer 4-vinylpyridine was grafted on polyethylene has been determined. The effect of radiation dose and film thickness on the amount of gel copolymer has been studied. Infrared and nitrogen analysis for the grafted films and the gel copolymer are given. Quaternium salts of the gel copolymer were prepared, and the melting point of the gel was determined. The insolubility of the gel copolymer was attributed to the probable formation of covalent-bond crosslinks between the grafted chains during irradiation.

KEY WORDS Gel / Copolymers / Radiation / Grafting / 4-Vinylpyridine / Polyethylene /
\end{abstract}

The gel formation following the radiation grafting on polymers of acrylic monomers such as acrylic and methacrylic acids, acrylonitrile, ${ }^{1}$ and acrylamide ${ }^{2}$ has been reported in the literatures. Campbell and Charlesby ${ }^{3}$ proposed that crosslinking of grafted polyethylene with acrylic acid occurs via a cationradical which forms on the polyethylene, while the graft copolymerization reaction is an ordinary free radical reaction; Singer et al. ${ }^{4}$ attributed the insolubility of the grafted poly(acrylic acid)polyethylene copolymer to a high degree of intermolecular hydrogen-bonding followed by the formation of covalent anhydride bonds. They performed analytical measurements to determine the gel content by xylene extraction at $130^{\circ} \mathrm{C}$ and elasticity measurements at $150^{\circ} \mathrm{C}$. The presence of these covalent bonds was confirmed by the infrared absorption spectrum.

Joshi, Silverman, and Singer ${ }^{1}$ studied the gel formation in the radiation grafting of acrylonitrile to polyethylene. The authors attributed the formation of covalent intermolecular links also to the postirradiation heat treatments used during coventional measurements of gel formation.

In a previous publication, ${ }^{5}$ we have studied the effect of grafting of 4-vinylpyridine on polyethylene on the mechanical properties. The results obtained show a significant increase in tensile load when the polyethylene films were highly grafted, and a drastic decrease in elongation when the same films were highly or slightly grafted.

The aims of the present work are to determine the amount of the gel formed due to grafting and to study the effect of the initial film thickness on the gel formation. Quaternization of the pyridine's nitrogen in the gel copolymer was also studied.

\section{EXPERIMENTAL}

\section{Materials}

4-Vinylpyridine produced by Fluka AG Chemische Fabric, Switzerland, of purity $95 \%$ was used with no further purification. Xylene was a product of Chemicoke El-Tabin, Egypt, range of boiling $120-150^{\circ} \mathrm{C}$. Low-density polyethylene, a product of C.D.F. Chemei, France, in the form of pellets was moulded into sheets by the compression moulding process shown in our previous publication. ${ }^{5}$ Methyl alcohol was a product of El-Nasr Co. for Pharmaceutical Chemicals, bp $64.5-66.0^{\circ} \mathrm{C}$. Allyl bromide was the quality available for scientific uses from Prolabo, France. Copper foils were highpurity materials obtained from $\mathrm{BDH}$, England. $\mathrm{HCl}$ was a product of El-Nasr Co. for Pharmaceutical Chemicals. 


\section{Procedures}

Grafting experiments were carried out by the direct-radiation technique. The homopoly(4vinylpyridine) was washed with methanol. The grafting was carried out on polyethylene films of thicknesses 1.9, 1.16, 0.36, 0.09, and $0.05 \mathrm{~mm}$. Films of thicknesses 1.9 and $1.16 \mathrm{~mm}$ were prepared in our laboratory by the method of press moulding at $150^{\circ} \mathrm{C}$, and the films of other thicknesses were provided by the National Plastic Company, ElOmrania, Giza, Egypt. The optical melting point was determined and found to be $109^{\circ} \mathrm{C}$, which corresponds to a density between 0.9142 and $0.9225 .{ }^{6}$ In our previous publication ${ }^{5}$ the details of the grafting process were explained. After grafting, films were extracted with methanol and dried and the weight $\left(W_{\mathrm{g}}\right)$ was recorded. The gel content was determined by boiling the grafted films in xylene in order to extract the ungrafted polyethylene. The insoluble part, i.e., the gel copolymer, was taken out and heated with methanol to remove the homopoly(vinylpyridine), then dried and weighed $\left(W_{\mathrm{e}}\right)$. This extraction cycle with xylene and methanol was repeated till the weight became constant.

The extracted methanol solution was found to be free of homopoly(vinylpyridine), while the xylene solution contains some extractable ungrafted polyethylene. This was checked by first concentrating the extracted solutions, then adding large amounts of precipitating agents to each solution separately. It should be mentioned here that both solvents used (methanol and xylene) are considered to be nonsolvents for the grafted polymers.

The insolubility of the gel was confirmed by heating it with solvents such as DMF, pyridine, and benzyl alcohol. The weight after heating with solvents and drying was found to be the same as before.

Infrared spectra were carried out using a Pye Unicam-type SP1000 spectrometer. The range of measurement was between wavenumbers 625 and $3600 \mathrm{~cm}^{-1}$.

\section{RESULTS AND DISCUSSION}

\section{Effect of Film Thickness on Grafting}

Figure 1 shows the change in the grafting yield with film thickness at different doses. It is observed that the percent graft drastically decreases with the increase in film thickness.

The high grafting yield for thin films may be due to

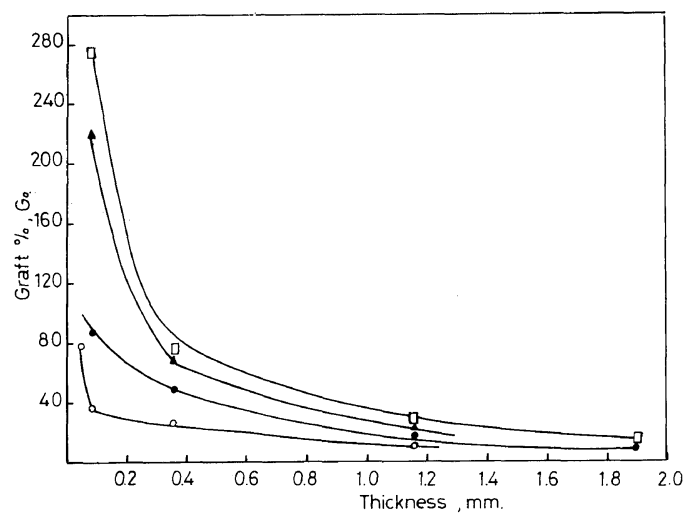

Figure 1. Graft extent vs. initial film thickness: $\bigcirc$, $0.263 \mathrm{Mrad}$; $0.526 \mathrm{Mrad}$, $\boldsymbol{\Delta}, 1.052 \mathrm{Mrad} ; \square, 1.315$ Mrad.

the fact that the grafting process within this range of film thickness could be regarded as a diffusion-free process. On the other hand, thick films yield a much lower grafting percentage. This could be due to the diffusion-controlled process of the monomer into the film. However, it was surprising to observe that the decrease in the graft yield with dose was more pronounced for thin films rather than for thick ones: e.g., for film thicknesses 0.1 to $0.4 \mathrm{~mm}$ the grafting yield decreases from 280 to $\sim 80 \%$ at the radiation dose of $1.3 \mathrm{Mrad}$, while for film thicknesses in the range $0.4-1.9 \mathrm{~mm}$, the grafting per cent decreases from 80 to $15 \%$. No direct explanation can now be given for such observations, but the decrease in graft yield may depend on the diffusion of the monomer through the bulk solution.

\section{Gel Determination}

The gel content was calculated as follows

$$
\mathrm{Gel} \%=\frac{W_{\mathrm{e}}}{W_{\mathrm{g}}} \times 100
$$

where $W_{\mathrm{g}}$ is the weight of the grafted film and $W_{\mathrm{e}}$ is the weight of the gel copolymer after extraction of the grafted film with xylene. The polyethylene (PE) content in these gel copolymers was calculated from the following equation

$$
\mathrm{PE} \%=\frac{W_{\mathrm{e}}-\left(W_{\mathrm{g}}-W_{\mathrm{o}}\right)}{W_{\mathrm{e}}} \times 100
$$

where $W_{\mathrm{o}}$ is the weight of the initial ungrafted polyethylene film.

Figure 2 shows the change in the gel content with 
Gel Formation in Grafting of 4-Vinylpyridine to Polyethylene

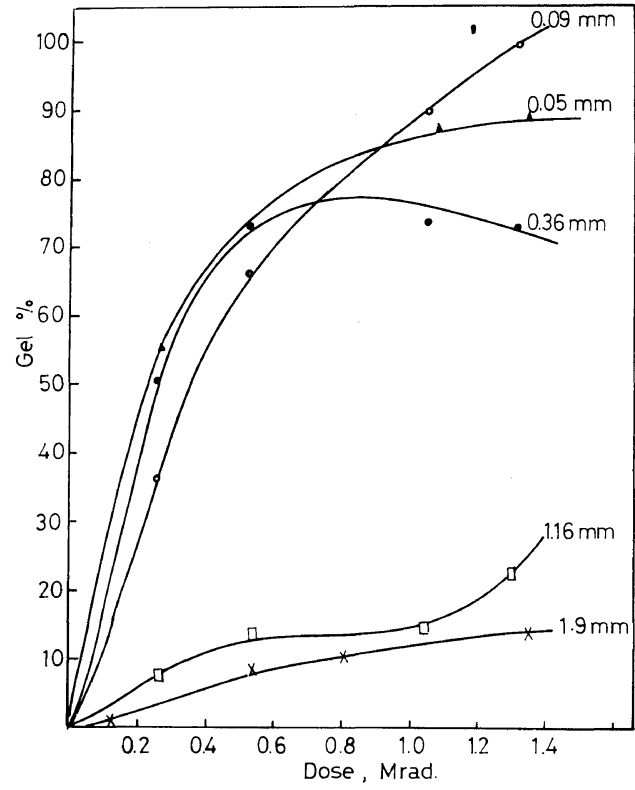

Figure 2. Gel content vs. radiation dose.

dose for different film thicknesses. It is observed from the figure that thin films $(0.05,0.09$, and $0.36 \mathrm{~mm}$ ) gave much higher gel copolymer yields than the thicker ones $(1.16$ and $1.9 \mathrm{~mm})$. The rate of change of the gel content for thin films was higher than for thick ones. This could be simply explained by noting that thin films had higher grafting percentages and consequently a higher gel content than the thicker ones.

Figure 3 shows the composition of the gel copolymer for the different film thicknesses used at the specified doses. It is observed that relatively thick films $(1.16$ and $1.9 \mathrm{~mm})$ contained a high poly(vinylpyridine) (PVP) percentage, while films of $0.36 \mathrm{~mm}$ thickness contain almostly equal amounts of PVP and $\mathrm{PE}$ at an irradiation dose of $0.8 \mathrm{Mrad}$. As a matter of fact, this figure could be considered as an experimental guide for preparing PE-PVP gel copolymers with a specific composition.

\section{Quaternization of the Gel Copolymer}

Quaternization of the gel grafted copolymer was carried out in order to prepare semipermeable membranes. Homogeneous strong basic membranes with mechanical strength have already been prepared from grafted copolymers of polyethylene-4vinylpyridine after being quaternized with methyl

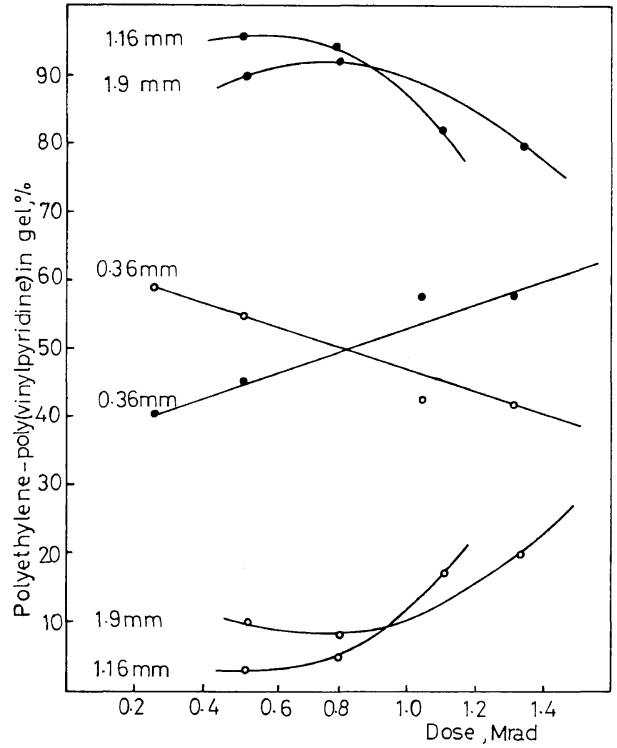

Figure 3. Gel composition at different doses: poly(vinylpyridine); $\bigcirc$, polyethylene. Dose rate, 75 $\operatorname{rads}^{-1}$.

iodide. $^{7}$

Polyethylene-poly(vinylpyridine) gel copolymer films, of weight $\left(W_{\mathrm{e}}\right)$, were immersed in allyl bromide or $\mathrm{CuCl}_{2}(\mathrm{Cu}$ metal $+\mathrm{HCl})$ and left overnight. The films were taken out, washed with methanol, dried, and weighed $\left(W_{\mathrm{q}}\right)$. Allyl bromide and $\mathrm{CuCl}_{2}$ contents $(Q)$ were calculated from the following equation

$$
Q(\%)=\frac{W_{\mathrm{q}}-W_{\mathrm{e}}}{\left(W_{\mathrm{q}}-W_{\mathrm{e}}\right)+W_{\mathrm{PVP}}} \times 100
$$

where $W_{\mathrm{PVP}}$ is the weight of poly(vinylpyridine) in the gel copolymer.

Theoretically, the interaction of one gram molecule of 4-vinylpyridine with one gram molecule of allyl bromide gives an increase of $53.5 \%$ in weight. Table I shows that the allyl bromide uptake by poly(vinylpyridine) is higher than the theoretically calculated $(53.5 \%)$ by $\sim 9 \%$. This increase in allyl bromide content may be partially due to some occluded unwashable allyl bromide in the film matrix.

It was surprising to observe that quaternization with $\mathrm{CuCl}_{2}$ leads to a lower content in the $\mathrm{CuCl}_{2}$ $(30.7 \%)$ than the theoretically calculated value $(39.00 \%)$. This may be due to the fact that the divalent $\mathrm{Cu}$ has to react with two pyridine groups, 
Table I. Variation of allyl bromide content with film thickness and dose

\begin{tabular}{|c|c|c|c|c|}
\hline Dose & $\begin{array}{c}\begin{array}{c}\text { Film } \\
\text { thickness }\end{array} \\
\end{array}$ & $\begin{array}{c}\text { PVP } \\
\text { content }\end{array}$ & \multirow{2}{*}{$\begin{array}{c}\text { Allyl } \\
\text { bromide } \\
\frac{Q}{\%}\end{array}$} & \multirow{2}{*}{$\begin{array}{c}\text { Deviation } \\
\text { from } \\
\text { theoretical }\end{array}$} \\
\hline Mrad & $\mathrm{mm}$ & $\%$ & & \\
\hline 0.26 & & 41 & 61.1 & 7.6 \\
\hline 1.05 & 0.36 & 57.5 & 61.1 & 7.6 \\
\hline 1.32 & & 57.7 & 62.5 & 9.0 \\
\hline 0.53 & & 69 & 65.9 & 12.4 \\
\hline 1.05 & 0.09 & 76 & 60.9 & 7.4 \\
\hline 1.32 & & 72 & 63.6 & 10.1 \\
\hline
\end{tabular}

which may not be easily available because of their fixed orientation in the gel copolymer.

\section{Nitrogen Analysis and PVP Content}

Table II shows the nitrogen content for the grafted films $(0.05 \mathrm{~mm}$ thickness) and their gel copolymers. The $C_{\mathrm{PVP}}$ content was calculated from the following equation

$$
\% C_{\mathrm{PVP}}=\frac{N}{14} \times 100
$$

where $N$ is the experimentally determined nitrogen value by the Dumas method in the grafted and the gel samples and the value 14 is the atomic weight of nitrogen.

From the Table, it is observed that $\% C_{\mathrm{PVP}}$ contents calculated from equations shown in the Table are equal to that calculated on the basis of nitrogen determination according to eq 1 which shows that the formed homopoly(vinylpyridine) is unextractable.

\section{Type of Bonding in the Gel Copolymer}

The insolubility of the gel copolymer could be attributed to the formation of intermolecular crosslinking via covalent bonds between the grafted as well as the mother polyethylene chains during irradiation.

\section{Infrared Analysis}

Infrared runs were completed for the grafted, gel, and mechanical mixture of polyethylene and poly(vinylpyridine). The mechanical mixture was prepared by spreading a thin layer of PVP, prepared by $\gamma$-radiation, over a thin film of low-density polyethylene.

Figure 4 shows the spectrum of a polyethylene-poly(vinylpyridine) graft copolymer gel. From the spectrum, it was observed that the characteristic bonds of both polyethylene and poly(vinylpyridine) appear clearly and almost without modification. A peak appearing at $1550 \mathrm{~cm}^{-1}$ confirms the existence of a nitroso $(\mathrm{N} \rightarrow \mathrm{O})$ group which could be attributed to the presence of oxidized nitrogen forms, since the irradiation grafting process was carried out in atmospheric oxygen. The appearence of these oxidized nitrogen forms in poly(vinylpyridine)s was also mentioned in the literature. ${ }^{8}$

An interesting observation for the characteristic band of the rocking polyethylene $-\mathrm{CH}_{2}-$ group, which appears clearly at $725 \mathrm{~cm}^{-1}$, was the gradual decrease in the intensity of absorption as the PVP content increases. This band vanishes completely in the gel copolymer after complete extraction of the ungrafted polyethylene portion in the grafted films.

The broadening and decrease in the intensity of absorption of the polyethylene $-\mathrm{CH}_{2}-$ group was due to the increase in the PVP content and the subsequent decrease of the PE content. This increase

Table II. Nitrogen analysis and $C_{\mathrm{PVP}}$ content

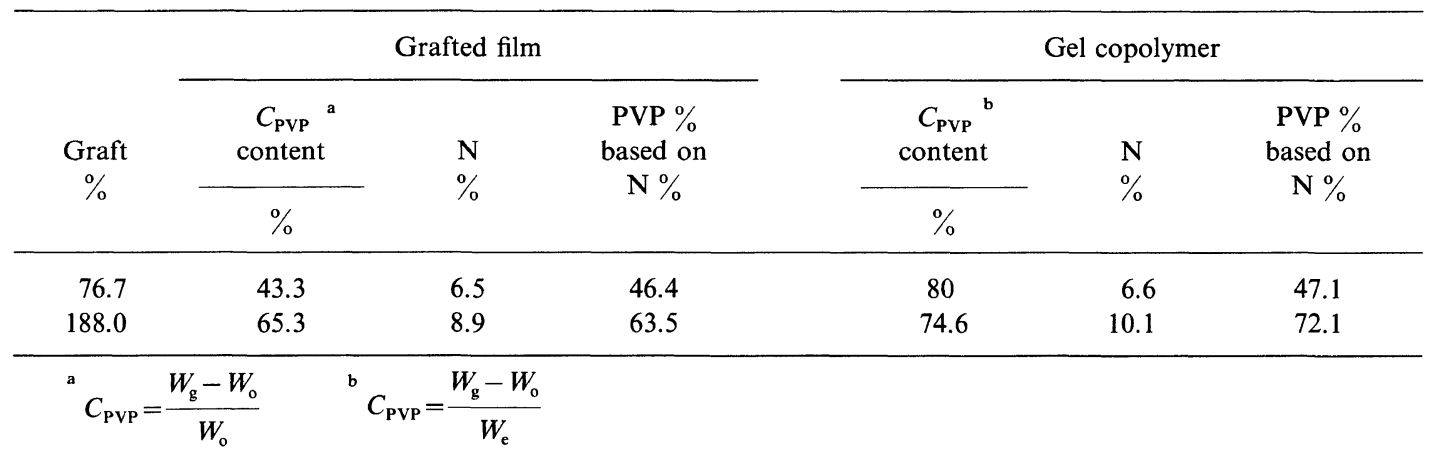


Gel Formation in Grafting of 4-Vinylpyridine to Polyethylene

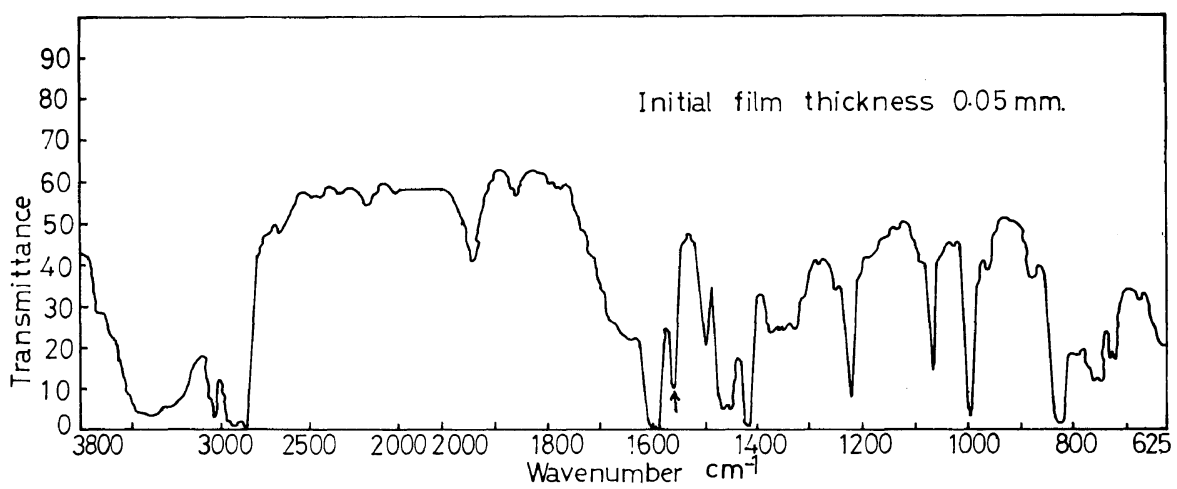

Figure 4. Infrared spectrum of polyethylene-g-poly(vinylpyridine). Poly(vinylpyridine) content, $70.5 \%$; polyethylene content, $29.5 \%$.

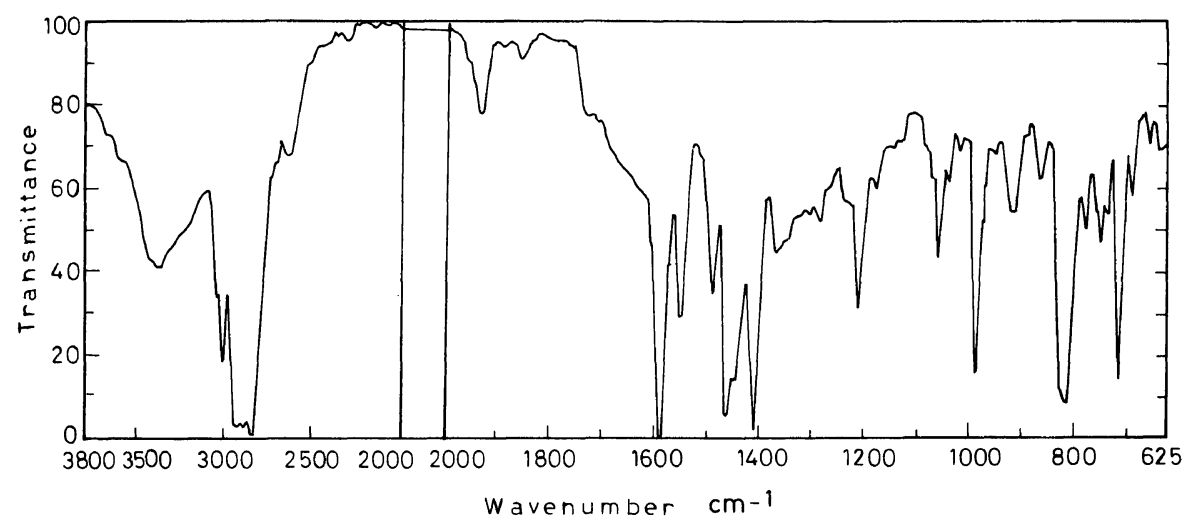

Figure 5. Infrared spectrum of the mechanical mixture of poly(vinylpyridine) and polyethylene.

in the PVP content may affect to a certain degree the physical structure of the grafted film, particularly the polyethylene $-\mathrm{CH}_{2}$ - rocking in the crystalline as well as the amorphous regions. The rest of the two spectra are almost the same, indicating that the gel copolymer does not contain any impurities or other functional groups.

It was found that the melting point of the grafted gel copolymer increased markedly $\left(269^{\circ} \mathrm{C}\right.$ for a gel copolymer with $75 \%$ PVP), when compared to the melting point of the initial ungrafted polyethylene $\left(109^{\circ} \mathrm{C}\right)$. This could be simply explained by the fact that the soft polyethylene chains were crosslinked by rigid poly(vinylpyridine) via hydrogen bonding. Poly(2-vinylpyridine) was shown to possess a relatively high melting point $\left(212^{\circ} \mathrm{C}\right) .^{9}$

\section{REFERENCES}

1. M. Joshi, J. Silverman, and K. Singer, J. Polym. Sci.,
Polym. Lett. Ed., 14, 723 (1976).

2. N. I. El-Awady, unpublished work.

3. D. Campbell and A. Charlesby, Chem. Zvesti, 26, 256 (1972).

4. K. Singer, M. Joshi, and J. Silverman, J. Polym. Sci., Polym. Lett. Ed., 12, 387 (1974).

5. A. M. Rabie, M. I. Aly, S. A. Hegazy, and N. I. ElAwady, Polym. J., accepted for publication.

6. J. Brandrup and E. H. Immergut, Ed., "Polymer Handbook", Interscience Publishers, New York, N.Y., 1967, VI 50.

7. M. Yanagita, H. Kawabe, K. Shinohara, and T. Takamatsu, The Scientific Papers of the Institute for Physical and Chemical Research, Tokyo, 56, 218 (1962); Chem. Abstr., 58, 1593 (1963).

8. D. O. Hummel, "Infrared Analysis of Polymers, Resins, and Additives, An Atlas," Vol. 1, Part 2, Wiley Interscience, London, 1969.

9. J. Brandrup and E. H. Immergut, Ed., "Polymer Handbook," Interscience Publishers, New York, N.Y., 1967, III 49. 